\title{
The Impact of Exposure to Liver
}

\section{Transplantation Anesthesia on the Ability to Treat Intraoperative Hyperkalemia: A Simulation Experience}

\author{
Dung Nguyen, Shira Gurvitz-Gambrel, Paul A. Sloan, Jeremy S. Dority, Amy DiLorenzo, \\ Zaki-Udin Hassan, Annette Rebel \\ Department of Anesthesiology, University of Kentucky, Lexington, Kentucky, USA
}

\begin{abstract}
The objective of this study was to assess whether resident exposure to liver transplantation anesthesia results in improved patient care during a simulated critical care scenario. Our hypothesis was that anesthesia residents exposed to liver transplantation anesthesia care would be able to identify and treat a simulated hyperkalemic crisis after reperfusion more appropriately than residents who have not been involved in liver transplantation anesthesia care. Participation in liver transplantation anesthesia is not a mandatory component of the curriculum of anesthesiology training programs in the United States. It is unclear whether exposure to liver transplantation anesthesia is beneficial for skill set development. A high-fidelity human patient simulation scenario was developed. Times for administration of epinephrine, calcium chloride, and secondary hyperkalemia treatment were recorded. A total of 25 residents with similar training levels participated: 13 residents had previous liver transplantation experience (OLT), whereas 12 residents had not been previously exposed to liver transplantations (non-OLT). The OLT group performed better in recognizing and treating the hyperkalemic crisis than the non-OLT group. Pharmacologic therapy for hyperkalemia was given earlier (OLT $53.3 \pm 27.0$ seconds versus non-OLT $148 \pm 104.1$ seconds; $P<0.01$ ) and hemodynamics restored quicker (OLT $87.9 \pm 24.9$ seconds versus non-OLT $219.9 \pm 87.1$ seconds; $P<0.01$ ). Simulation-based assessment of clinical skills is a useful tool for evaluating anesthesia resident performance during an intraoperative crisis situation related to liver transplantations. Previous liver transplantation experience
\end{abstract}

Corresponding author: Annette Rebel, MD, Department of Anesthesiology, University of Kentucky Medical Center, 800 Rose Street, Lexington, KY 40536.

Tel.: 859323 5956; Fax: 859323 1080; E-mail: arebe2@email.uky.edu 


\section{improves the anesthesia resident's ability to recognize and treat hyperkalemic cardiac arrest.}

Key words: Liver transplant - Anesthesia - Hyperkalemia - Reperfusion - Simulation Crisis management - Resident education

$\mathrm{A}$ nesthesia care during orthotopic liver transplantation can be very challenging because of the complexity of altered physiology secondary to end-stage liver disease, combined with a very complex surgical procedure causing hemodynamic instability secondary to blood and volume shifts. Although providing anesthesia care during orthotopic liver transplantation exposes anesthesiology residents to several unique challenges, not all anesthesiology training programs expose their residents to these kinds of cases; nor is there a requirement by the American Board of Anesthesiology to do so. ${ }^{1}$ A recent simulation-based project indicated that simulation training in intraoperative liver transplantation anesthesia care improved a resident's ability to provide anesthesia care during these situations. ${ }^{2}$ Several other publications have indicated that simulation training improves skill level and the ability to communicate in crisis situations. $^{3-5}$ However, it is unclear whether the exposure to high-complexity anesthesia care (e.g., cardiothoracic anesthesia, liver transplantation anesthesia) will improve a resident's overall crisis management skills. There is evidence from surgical specialties that duty hour restrictions reduced the time for education. ${ }^{6,7}$ In surgical subspecialties using complex technology, traditional teaching methods could not keep up with the current need for knowledge and skill acquisition. ${ }^{8}$ Because residency training time in the operating room has decreased in recent years because of work hour limitations as well as the need to expose residents to new techniques (e.g., transesophageal echocardiography, peripheral nerve blocks, critical care), anesthesiology residency program directors may have to choose which rotations provide the maximal learning benefit for the residents to develop their skill set. To answer the question of whether training in liver transplantation anesthesia care is advantageous for all residents, we designed a human patient simulation project. Our hypothesis was that anesthesia residents exposed to liver transplantation anesthesia care would be able to identify and treat a simulated hyperkalemic crisis after reperfusion more appropriately and in a more timely manner than residents who have not been involved in liver transplantation anesthesia care.

\section{Methods}

After institutional review board approval, anesthesiology residents eligible for liver transplantation anesthesia (postgraduate years 3-4) were asked to participate. By our departmental standards, the resident has to have his or her first cardiothoracic anesthesia rotation completed to be eligible for transplantation call. The residents in the liver transplantation experience group (OLT) had participated in at least one liver transplantation, whereas residents in the non-OLT group were eligible for OLT call but had not participated in any liver transplantation procedures at the time of the simulation project. The residents in the OLT group had participated in 1 to 4 liver transplantations, whereas residents with more than 4 liver transplantations were excluded from the study to maintain a training level with similar to that of the non-OLT residents. A patient simulator scenario was developed by the authors for this project. After the resident completed the simulation scenario, the video recordings were assessed and evaluated by a faculty member blinded to the liver transplantation experience of the participant.

\section{Patient scenario}

This scenario was conducted within the patient simulation laboratory at the University of Kentucky, using a patient simulator (Standard Man, 2000, Medical Educational Technologies Inc, Sarasota, Florida), a standard Phillips patient monitor (Phillips Medical Systems North America, Hoffman Estates, Illinois), and a mainstream anesthesia gas machine with oxygen/nitrous oxide fresh gas supply. Physiologic parameters, including arterial blood pressure, heart rate, pulse oxymetry, capnography, and central venous and pulmonary artery pressures, are continuously displayed during the scenario. In the scenario, the resident leads an operating room team during the reperfusion stage of an orthotopic liver transplantation. Hyperkalemia occurs after reopening of 
Fig. 1 Simulation scenario timeline.

the portal vein, followed by hemodynamic deterioration progressing to cardiac arrest. While the surgeon initiates CPR, the anesthesia resident is expected to treat the hyperkalemic crisis. The time frame for the events during the scenario with expected interventions is shown in Fig. 1.

Times for administration of epinephrine (epinephrine), calcium chloride (calcium), and secondary hyperkalemia treatment [insulin/glucose, sodium bicarbonate, or albuterol (Hyperkalemia Tx)] are recorded. After a minimum of 2 hyperkalemia treatments have been given, the simulation scenario resumes hemodynamic stability with sinus tachycardia. Time to hemodynamic stabilization (HD restored) is recorded. If the resident fails to identify and address the situation, or if incorrect actions are initiated, the patient's condition deteriorates further and the simulation scenario ends (end point $=10$ minutes of CPR, recorded as 600 seconds).

Video recording was used for the assessment of the crisis management and recording of the time to intervention. Times are given in seconds, starting at the beginning of reperfusion.

For the data analysis, participating residents were separated into two groups: residents with liver transplantation experience (OLT, $\mathrm{n}=13$ ), and residents without liver transplantation experience (non-OLT, $\mathrm{n}=12$ ). All residents had similar exposure to cardiothoracic and general anesthesia, as well as critical care medicine. Therefore, the residents' training levels appeared to be similarly distributed amongst the two groups, except for the liver transplantation experience. Data are presented by mean \pm SD. Statistical analysis was performed by the unpaired $t$-test as a parametric test and the Mann-Whitney $U$ test as a nonparametric test without assumption about probability distribution. Statistical significance was assumed if $P<0.05$.

Once the simulation experience and debriefing concluded, the participating residents were asked to rate their simulation experience and value for their development using 5 Likert-type scale questions.

\section{Results}

A total of 25 residents in the training years of postgraduate years 3 to 4 participated in the study. A total of 12 residents had no previous exposure to liver transplantation anesthesia care (non-OLT, $\mathrm{n}=$ 12), whereas 13 residents had experience with liver transplantation anesthesia care prior to the simulation scenario (OLT, $\mathrm{n}=13$ ). Times to epinephrine, calcium chloride, and hyperkalemia treatment are shown in Table 1. Appropriate pharmacologic therapy in response to the hemodynamic deterioration associated with hyperkalemia was initiated earlier by residents with previous liver transplantation anesthesia training (OLT) compared with residents without liver transplantation training (non-OLT). Only 1 resident in the non-OLT group failed to recognize the hyperkalemic crisis and did not initiate appropriate treatment.

Hemodynamic restoration occurred earlier in the OLT group compared with the non-OLT group [87.9 \pm 24.9 seconds versus $219.9 \pm 87.1$ seconds, respectively (Fig. 2)]. 
Table 1 Time to pharmacologic therapy ${ }^{\mathrm{a}}$

\begin{tabular}{lcccc}
\hline & Non-OLT & OLT & $P$ value $t$-test & $P$ value Mann-Whitney $U$ test \\
\hline Epinephrine & $73.8 \pm 13.3 \mathrm{~s}$ & $49.9 \pm 10.1 \mathrm{~s}^{\mathrm{b}}$ & 0.0003 & 0.00016 \\
Calcium & $158.5 \pm 103.2 \mathrm{~s}$ & $51.5 \pm 28.2 \mathrm{~s}^{\mathrm{b}}$ & 0.0015 & 0.00076 \\
Hyperkalemia therapy & $148.0 \pm 104.1 \mathrm{~s}$ & $53.3 \pm 27.0 \mathrm{~s}^{\mathrm{b}}$ & 0.0043 & 0.00228 \\
\hline
\end{tabular}

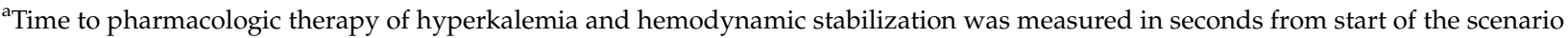
(reperfusion) to the recorded intervention: Epinephrine, calcium chloride and hyperkalemia therapy (minimum of 2 items: insulin/ glucose; albuterol; sodium bicarbonate; hyperventilation). Non-OLT residents $(n=12)$, OLT residents $(n=13)$. Data are shown as mean $\pm \mathrm{SD}$.

${ }^{\mathrm{b}} P<0.01 . P$ value per unpaired $t$-test (parametric) and Mann-Whitney $U$ test (nonparametric).

Resident evaluations indicated that all residents (OLT and non-OLT) found the experience valuable and would recommend the experience to others (Fig. 3).
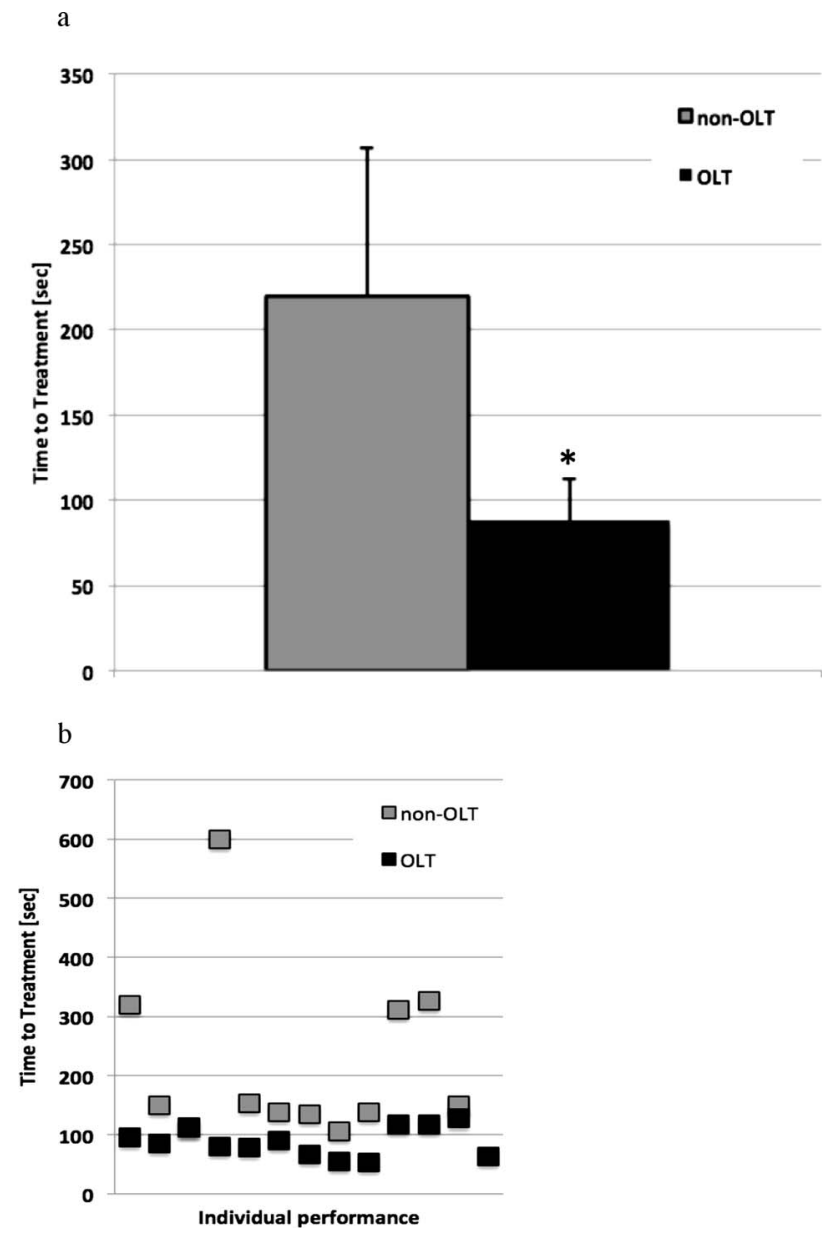

Fig. 2 Time to hemodynamic restoration was measured in seconds from start of the scenario (reperfusion) to the return of spontaneous circulation after hyperkalemic cardiac arrest. NonOLT residents $(n=12)$, OLT residents $(n=13)$. (a) Data are shown in Fig. 3a as mean \pm SD. ${ }^{*} P=0.0007$ (unpaired $t$-test) and $P=$ 0.00012 (Mann-Whitney $U$ test). (b) Individual distribution.

\section{Discussion}

The presented data indicate that residents actively involved in anesthesia care during a liver transplantation respond faster to a simulated hyperkalemic crisis than residents who have not been previously involved in liver transplantation anesthesia.

Recent reviews have summarized the value of simulation training during anesthesiology residency and postcertification training. ${ }^{9-12}$ However, most of these publications emphasize the advantage of simulation training in improving patient care. Anesthesia residents have been shown to perform better during emergencies if they received prior simulation training. ${ }^{13,14}$ Our study used simulation to investigate the reverse relationship. Based on our observations, clinical exposure to liver transplantation anesthesia improved the anesthesia provider's ability to manage a mannequin-based simulation crisis.

Although Aggarwal et $a l^{2}$ have shown that liver transplantation anesthesia care can be taught in a simulation-based setting, our project provides evidence that the clinical exposure to liver transplantation anesthesia care is beneficial for a resident's development of his or her overall ability of crisis management. We have chosen a hyperkalemic crisis because the situation is a common occurrence during liver reperfusion but also can occur during routine anesthesia care. Hyperkalemic crisis is not exclusively linked to intraoperative liver transplantation care, and the ability to effectively manage a hyperkalemic crisis should be expected of all anesthesia providers. It is interesting that only a limited exposure to liver transplantation anesthesia improved the crisis management skills of residents included in our study. Previous studies in surgical residents have indicated that it may take several training experiences to improve performance, depending on the complexity of the learned task. ${ }^{15,16}$ The difference with our findings might be explained 
Post Scenario Survey
Fig. 3 Using a survey of all participants $(\mathrm{n}=25)$ after the simulation experience, the perception of the learning experience was rated using a Likert scale, with 1 being strongly disagree and 5 being strongly agree (as shown in Fig. 1). Data are shown as mean $\pm \mathrm{SD}$.

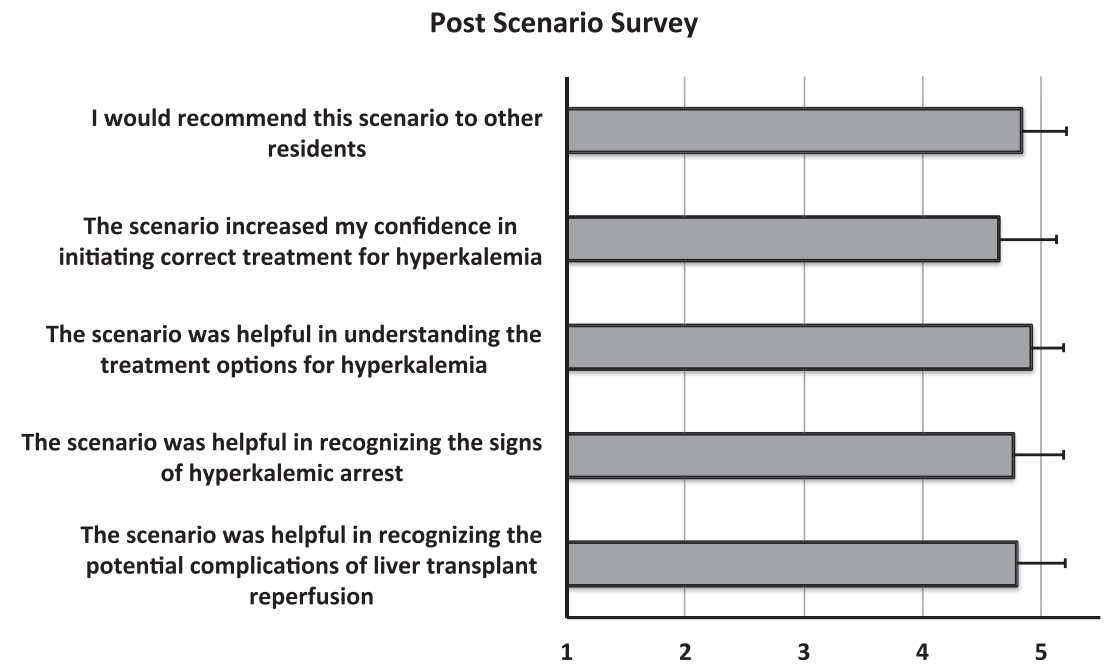

by the intense educational learning with each liver transplantation experience. The case is staffed with a 1:1 attending-to-resident ratio, and detailed actions for possible hyperkalemia crisis are discussed before every reperfusion. Unfortunately, the presented study is not powered enough to provide more detailed information regarding a correlation between crisis management skills and frequency of exposure to liver transplantation anesthesia. Additional research should address this question.

All residency programs have been challenged by the Accreditation Council for Graduate Medical Education to document residents' skill progression during their residency through the assessment and reporting of Milestones. ${ }^{17}$ The ability to respond to and manage crisis situations in a timely manner is essential for the field of anesthesiology. However, this skill set is very difficult to teach and assess in clinical practice. Simulation can be used to objectively assess the ability to provide patient care during crisis situations. ${ }^{4,18}$ Recording of the scenario can be used for debriefing and documentation. We found that simulation-based assessment is valid to document a difference in clinical practice depending on the resident's training level. Therefore, simulation should be seen as a valuable tool to assess skill progression and Milestone competency during the anesthesiology residency.

Concerns have been expressed about using clinical simulation for assessment of competency because case specificity may pose a noticeable threat to the reliability and validity of the assessment. ${ }^{19}$ We chose a relatively short simulation scenario with a very defined clinical problem. However, because this is a very specific situation to be addressed, the findings may not be generalizable toward a broader clinical skill assessment. To be specific: the ability to recognize and treat a hyperkalemic crisis related to reperfusion during liver transplantation does not guarantee that the resident is able to treat the same way in a situation not related to liver transplantation, or that the resident is able to treat other intraoperative complications related to events during liver transplantation.

The ability of simulation to assess clinical performance has been previously investigated. ${ }^{20,21}$ The multiscenario approach was more reliable than that involving one scenario. ${ }^{18}$ Although our study confirms the findings of previous studies, the interpretation of our findings is limited because we only investigated a single liver anesthesia-related crisis situation. Therefore, the beneficial impact of liver transplantation anesthesia on crisis management skills should be confirmed by additional simulation-based evaluations. Another limitation of the interpretation of our findings is the single-institution-based study design. Systems-based practice and awareness of institutional resources are essential for the success of crisis management. Therefore, the described approach to treatment of hyperkalemic crisis after liver transplantation reperfusion might not be directly transferable to other institutions. However, the simulation-based practice might give the residents an opportunity to develop their communication and leadership skills.

In anesthesiology, simulation has been introduced as a component of the primary certification and recertification process of the American Board of Anesthesiology. As outlined by Boulet and Murray, ${ }^{22}$ this process is not without contention, and more validity evidence has been requested. This study adds evidence to the validity of simulation as an assessment option for clinical competence. 
Our project did not address whether the clinical exposure to liver transplantation anesthesia is superior to other training methods for improving crisis management skills. Additional investigations are needed to explore whether other didactic techniques would have an impact on crisis management skills that was similar to that of clinical experience. Future investigations should expand the simulation scenario to include management of other liver transplantation complications during reperfusion.

In summary, our findings support the use of simulation-based assessment of clinical skills for evaluating anesthesia resident performance during an intraoperative crisis related to liver transplantation. Previous liver transplantation experience improved the anesthesia resident's ability to recognize and treat hyperkalemic cardiac arrest. These findings support the value of clinical exposure to liver transplantation anesthesia care for the development of crisis management skills.

\section{References}

1. American Board of Anesthesiology. Residency program requirements. Available at: https:/ / www.acgme.org/acgmeweb / tabid/128/ProgramandInstitutionalAccreditation/HospitalBasedSpecialties/Anesthesiology.aspx. Accessed June 3, 2014

2. Aggarwal S, Bane BC, Boucek CD, Planinsic RM, Lutz JM, Metro DG. Simulation: a teaching tool for liver transplantation anesthesiology. Clin Transplant 2012;26(4):564-570

3. Yee B, Naik VN, Joo HS, Savoidelli GL, Chung DY, Houston PL et al. Nontechnical skills in anesthesia crisis management with repeated exposure to simulation-based education. Anesthesiology 2005;103(2):241-248

4. Murray DJ, Boulet JR, Kras JF, McAllister JD, Cox TE. A simulation-based acute skills performance assessment for anesthesia training. Anesth Analg 2005;101(4):1127-1134

5. Hassan ZU, Sloan PA. Using a mannequin-based simulator for anesthesia resident training in cardiac anesthesia. Simul Healthc 2006;1(1):44

6. Vanderveen K, Chen M, Scherer L. Effects of resident dutyhours restrictions on surgical and nonsurgical teaching faculty. Arch Surg 2007;142(8):759-766

7. Ahmed N, Devitt KS, Keshet I, Spicer J, Imrie K, Feldman L et al. A systematic review of the effects of resident duty hour restrictions in surgery: impact on resident wellness, training, and patient outcomes. Ann Surg 2014;259(6):1041-1053

8. Antonoff MB, Verrier ED, Yang SC, Lin J, DeArmond DT, Allen MS et al. Online learning in thoracic surgical training: promising results of multi-institutional pilot study. Ann Thorac Surg 2014;98(3):1057-1063

9. Rebel A., Hatton KW, Sloan PA, Hayes CT, Sardam SC, Dority $\mathrm{J}$ et al. Neurophysiological monitoring simulation using flash animation for anesthesia resident training. Simul Healthc. 2011; 6(1):48-54

10. LeBlanc VR. Simulation in anesthesia: state of the science and looking forward. Can J Anaesth 2012;59(2):193-202

11. Naik VN, Brien SE. Simulation: a means to address and improve patient safety. Can J Anaesth 2013;60(2):192-200

12. Park CS. Simulation and quality improvement in anesthesiology. Anesthesiol Clin 2011;29(1):13-28

13. Schwid HA, Rooke GA, Michalowski P, Ross BK. Screen-based anesthesia simulation with debriefing improves performance in a mannequin-based anesthesia simulator. Teach Learn Med 2001;13(2):92-96

14. Kuduvalli PM, Jervis A, Tighe SQ, Robin NM. Unanticipated difficult airway management in anaesthetized patients: a prospective study of the effect of mannequin training on management strategies and skill retention. Anaesthesia 2008; 63(4):364-369

15. Tavakol M, Mohagheghi MA, Dennick R. Assessing the skills of surgical residents using simulation. J Surg Educ 2008;65(2): 77-83

16. Dawe SR, Windsor JA, Broeders JA, Cregan PC, Hewett PJ, Maddern GJ. A systematic review of surgical skills transfer after simulation-based training. Ann Surg 2014;259(2):236-248

17. Accreditation Council for Graduate Medical Education. ACGME program accreditation requirements for anesthesiology. Available at: https://www.acgme.org/acgmeweb/ tabid/128/ProgramandInstitutionalAccreditation/HospitalBasedSpecialties/Anesthesiology.aspx. Accessed June 3, 2014

18. Murray DJ, Boulet JR, Avidan M, Kras JF, Henrichs B, Woodhouse $\mathrm{J}$ et al. Performance of residents and anesthesiologists in a simulation-based skill assessment. Anesthesiology 2007;107(5):705-713

19. Schuwirth LW, Van der Vleuten CP. The use of clinical simulation in assessment. Med Educ 2003;37(suppl 1):65-71

20. Devitt JH, Kurrek MM, Cohen MM, Fish K, Fish P, Noel AG et al. Testing internal consistency and construct validity during evaluation of performance in a patient simulator. Anesth Analg 1998;86(6):1160-1164

21. Schwid HA, Rooke GA, Carline J, Steadman RH, Murray WB, Olympio $\mathrm{M}$ et al. Evaluation of anesthesia residents using mannequin-based simulation: a multiinstitutional study. Anesthesiology 2002;97(6):1434-1444

22. Boulet JR, Murray DJ. Simulation-based assessment in anesthesiology: requirements for practical implementation. Anesthesiology 2010;112(4):1041-1052

(C) 2015 Nguyen et al.; licensee The International College of Surgeons. This is an Open Access article distributed under the terms of the Creative Commons Attribution Non-commercial License, which permits use, distribution, and reproduction in any medium, provided the original work is properly cited, the use is non-commercial and is otherwise in compliance with the license. See: http://creativecommons.org/licenses/by-nc/3.0 